\title{
Diabetics Under Report Energy Intake in NHANES III Greater than Non- Diabetics
}

\author{
Michael E. Broyles, Robin Harris and Douglas L. Taren* \\ Mel and Enid Zuckerman College of Public Health, University of Arizona, Tucson, Arizona, USA
}

\begin{abstract}
Known factors associated with under-reporting energy intake (EI) include anthropometry, energy expenditure, psychological factors, smoking, and gender. There is insufficient information on the association between chronic disease status and under-reporting EI. Using NHANES III data from non-pregnant adults aged $\geq 20$ years, reported EI was estimated from a single 24-hour dietary recall and compared with estimated basal metabolic rate $\left(\mathrm{BMR}_{\mathrm{est}}\right)$ among 1503 diabetics and 17,010 non-diabetics. Multiple linear regression and logistic regression were performed to compare underreporting between diabetics and non-diabetics, and if it was associated with a hemoglobin A1c within diabetics.

Using EI:BMR $\mathrm{est}_{\mathrm{e}}<0.9$, male and female diabetics were 2.1 and 3.4 times as likely to under report EI compared to nondiabetics after controlling for weight, age, education, usual intake level, physical activity, tobacco and alcohol use. This greater under-reporting by diabetics could bias the association between diet and disease toward the null in epidemiological studies.
\end{abstract}

Keywords: Dietary methods, 24-hour recalls, Energy intake, Diabetes, NHANES, Bias, Epidemiology.

\section{INTRODUCTION}

Understanding the effects of bias due to under-reporting is important in studies of diet-disease relationships. Accounting for potential under-reporting in studies of diet-disease relationships can be relatively simple if the proportion of food constituents under-reported is in proportion to total energy intake (EI) under-reported. Although considerable research has been done correlating dietary under-reporting with behavioral, socio-demographic and anthropometrics values, little research has been reported connecting dietary under-reporting with specific chronic diseases.

Comparing reported EI with estimate of energy expenditure is the usual way to assess under-reporting. Typical measures of energy expenditure include estimated basal metabolic rate $\left(\mathrm{BMR}_{\mathrm{est}}\right)$, with or without adjusting for physical activity [1-5], whole- body calorimetric [6], doubly labeled water [6-8], and urinary biomarkers [9-10]. Though they may yield more accurate estimates, calculating energy expenditure via calorimetric, doubly labeled water and urinary biomarkers is expensive and may be impractical for studies of large samples.

Regardless of the method chosen to estimate energy expenditure and irrespective of the method used to assess diet, under-reporting of EI is considered to be widespread. Briefel et al. [2] used a single 24-hour recall estimate of EI compared with $\mathrm{BMR}_{\text {est }}$ to describe under-reporting in the first phase of the Third National Health and Nutrition Examination Survey (NHANES III), with data collected between 1988 and 1991. Under-reporting was more likely to occur in

*Address correspondence to this author at the Mel and Enid Zuckerman College of Public Health, University of Arizona, 1295 North Martin Ave, PO Box 245163, Tucson, Arizona 85724, USA;

E-mail: taren@email.arizona.edu women than men, persons who were older, and overweight individuals as defined by body mass index (BMI) and in people attempting to lose weight. It also varied by smoking status, educational attainment and physical activity. These results were similar to those obtained in national surveys in Norway [3] and Britain [4], which used food-frequency questionnaires and weighed 7-day intake methods, respectively, to determine EI. Other factors reported to influence dietary under-reporting include socioeconomic status $[2,4$, 8], literacy [8], health consciousness [3], percent body fat [11], and current or recent illness [2].

Diabetes mellitus is a condition chiefly managed through dietary and lifestyle changes. In 1998, the prevalence of diabetes in the United States (U.S.) rose to $6.5 \%$, a $33 \%$ increase from 1990 [12]. Increasing rates of obesity, diabetes are expected to become even more common [13-14]. Worldwide, between 1995 and 2025, a rise in diabetes prevalence of $42 \%$ is predicted for industrialized countries and $170 \%$ for developing nations [15]. Diabetics who do not control their glucose levels, as measured by hemoglobin A1c (HbA1c) concentrations, experience more severe complications, including kidney disease, retinopathy, stroke, and ischemic heart disease $[14,16]$. In addition to severe physical burden, diabetes carries a heavy financial cost. Nearly $15 \%$ of all U.S. health care dollars are spent on patients with diabetes, a figure likely to rise with the increase in diabetes prevalence [17].

Data examining the relationship between under-reporting EI and diabetic status are limited. One small study found under-reporting more prevalent among young type 1 diabetics [18]. No large studies have examined if an association between diabetic status and under-reporting exists.

The current research was conducted using data from NHANES III to examine if an association between self- 
identified diabetic status and dietary under-reporting exists and to evaluate how other demographic and behavioral factors impact the relationship. Furthermore, because NHANES data are used to formulate national health and nutrition policy, this research sought to determine if under-reporting was associated with long-term glycemic control in self-identified diabetics.

\section{MATERIALS AND METHODOLOGY}

\section{Survey Design and Variables Assessed}

NHANES III was designed as a two-phase, six-year survey to be representative of the civilian, non-institutionalized U.S. population [19]. Blacks, Hispanics, children aged 2 months to 5 years, and persons aged 60 years and over were over sampled, ensuring sufficient samples to be representative of each sociodemographic group. Household interviews were combined with physical examinations conducted at mobile examination centers throughout the U.S. Interviews assessed past and present lifestyle practices including physical activity, tobacco and alcohol use and other factors. A full physical examination was performed, including assessment of body measurements, blood and urine tests and completion of a 24-hour dietary recall. Specifics of the assessment are described elsewhere $[2,19]$.

The dataset was contained in a two-disc CD-ROM set obtained from the National Center for Health Statistics. Full documentation regarding data collected and appropriate analysis methods were also included [19].

Data used in the current analyses included information from the 24-hour dietary recall, diabetic status, measured height and weight, age in years, years of education completed, income level, race-ethnicity, and information on tobacco and alcohol use, "usual" intake level, physical activity, birthplace, income level, recent illness, medication use, recall day, and blood values.

Diabetic status was based on participant self-report. Height and weight assessment procedures have been described elsewhere [2]. Body mass index (BMI) was calculated as $\mathrm{kg} / \mathrm{m}^{2}$. Individuals were classified as underweight (BMI < 18.5), normal weight (BMI 18.5 to 24.9), overweight (BMI 25.0-29.9), and obese (BMI $\geq 30.0$ ). Desire to maintain, increase, or decrease current weight, and currently trying to lose weight were assessed via questionnaire.

Income level was measured using a poverty-income ratio, derived from family income divided by the federal poverty threshold, adjusting for family size. A poverty-income ratio less than 1 was classified as "below poverty." Race-ethnicity was determined by individual self-description as nonHispanic white, non-Hispanic black, Hispanic or other. Birthplace was coded as U.S. $=0$ or Other $=1$. Recent illness was classified as the number of colds in the past 4 weeks.

Tobacco use was categorized into cigarette use, cigar and pipe use, and chewing tobacco / snuff use. Cigarette use was divided into two variables: ever smoked 100 or more cigarettes and currently smoke cigarettes. Similarly, cigar and pipe use was defined as ever smoked 20 or more cigars or pipes of tobacco and currently smoke cigars or pipe tobacco. Chewing tobacco / snuff use was defined as ever used and currently use chewing tobacco or snuff. Alcohol use was defined as ever consumed 12 or more alcoholic drinks (yes/no), consumed 12 or more alcoholic drinks in the past year (yes/no), and then as energy from alcohol and percentage of total energy from alcohol estimated from the 24 hour recall. "Usual" intake was for the 24-hour recall and based on whether the previous day's intake was reported as being "more than usual", "usual," and "less than usual." Physical activity was classified by summing all leisure-time physical activity reported in the month preceding the interview. Monthly activity was also converted to average weekly physical activity and into weekly physical activity categories, "none", "1-2 times per week" and " 3 or more times per week." The participant's impression of his or her physical activity level compared to peers (equal, greater or less) was recorded by interview.

Medication use variables included antihistamine use in the past two days (yes/no), number of prescription medications taken in past month, and use of vitamins and minerals in the past 24 hours (yes/no). Diabetic medications were assessed as currently taking oral hypoglycemic medication (yes/no) and currently using insulin (yes/no). Blood values included fasting plasma glucose, plasma glucose following an oral glucose tolerance test, $\mathrm{HbAlc}$, serum insulin, and serum c-peptide. Procedures for collection and analysis of these are described elsewhere [19].

\section{Inclusion and Exclusion of Participants}

All persons aged 20 years and older were included in the analyses. All pregnant women were excluded based on selfreport or a positive urine test.

\section{Determination of Reporting Status}

To quantify degree of potential under-reporting of EI, the ratio of EI, estimated from the 24-hour recall versus estimated basal metabolic rate $\left(\mathrm{BMR}_{\text {est }}\right)$ was calculated. Basal metabolic rate $(\mathrm{kj} / \mathrm{day})$ was estimated with age and sexspecific formulas derived by Schofield [20] using the height and weight measured in the mobile examination center. Examples of formulas for persons aged 30 to 60 years are as follows:

\section{Males}

BMR $=0.048$ (weight, $\mathrm{kg}$ ) -0.011 (height, $\mathrm{m})+3.670$

\section{Females}

$\mathrm{BMR}=0.034$ (weight, $\mathrm{kg}$ ) +0.006 (height, $\mathrm{m})+3.530$

We used the cutoff value, of Briefel et al. [2] to classify individuals as under-reporters $\left(\mathrm{EI}: \mathrm{BMR}_{\mathrm{est}}\right.$ was $\left.<0.9\right)$. Those with a ratio of EI:BMR est $\geq 0.9$ were considered adequate reporters.

\section{Statistical Methods and Hypothesis Testing}

Data were weighted according to survey design to be representative of the national US population and to account for irregularities, such as participant non-response. All analyses were performed using Intercooled Stata 6.0 (Stata Corporation, College Station, TX), complex survey analysis procedures, and according to the weights, strata and population sampling units (PSU) included in the NHANES III data set. Results reported include population percentages, means and standard errors of the mean (SEM). Two-sided $t$ tests 
Table 1. Number of Self-Identified Diabetic and Non-Diabetic Participants in the Third National Health and Nutrition Examination Survey, 1988-1994

\begin{tabular}{|c|c|c|c|}
\hline Diabetes Status & Male & Female & Total n (\% of total) \\
\hline Self identified diabetic $\mathrm{n}$ (\% of diabetics) & $658(45.1 \%)$ & $845(54.9 \%)$ & $1503(5.2 \%)$ \\
\hline Total & $9705(48.4 \%)$ & $8808(51.6 \%)$ & $18513(100 \%)$ \\
\hline
\end{tabular}

${ }^{1}$ Number of participants shown, $n$, is the raw number of participants in the data set. Percentages shown are calculated employing survey weights, strata and PSUs and are the percentages of each category based on the population size represented in the data set; percentages shown may not equal those calculated on a raw basis.

were used to test for statistical differences between groups for continuous variables.

To determine the difference between diabetics and nondiabetics in regards under-reporting EI, we used a series of multiple logistic regressions stratified by men and women. Criteria for covariate inclusion into the models included $\mathrm{p}$ values of less than 0.05 and $p$ less than 0.10 or less for interaction terms. Potential inclusion was selected based on published results from similar studies and on bivariate analyses resulting in p-values less than 0.20 . These variables included overweight classification or body mass index, age, education, whether the reported diet was representative of the participant's "usual" intake, currently trying to lose weight, physical activity compared to peers, birthplace (U.S. vs. Other), poverty status, recent illness, tobacco use, alcohol use, recall day (weekend day vs. weekday), desire to increase, decrease or maintain current weight, and recent medication and vitamin/mineral usage. Self-identified diabetes status was forced into the models to determine if known diabetic state was associated with under-reporting.
Blood $\mathrm{HbA} 1 \mathrm{c}$ percentage was used as an indicator of long-term glycemic control among diabetics [21-22]. A series of multiple linear regressions were employed to determine if there was an association between blood HbAlc levels and under-reporting of EI among self-identified diabetics, stratified by gender. Multiple logistic regression analyses were also used to determine if clinically high versus normal blood $\mathrm{HbA} 1 \mathrm{c}$ percentage differed between diabetics who under-reported EI and those who did not under-report EI. Clinically high and normal $\mathrm{HbAlc}$ values were classified according to the criteria of the National Institute of Diabetes and Digestive and Kidney Diseases. Individuals were defined to have a high $\mathrm{HbA1c}$ blood percentage with $\mathrm{HbA1c}$ values $>7 \%$, while those with $\mathrm{HbA} 1 \mathrm{c} \leq 7 \%$ were considered to have normal HbAlc levels. Variables were considered for entry into models based on $\mathrm{p}<0.05$ and interaction terms based on $\mathrm{p}<0.10$. Variables included serum c-peptide level, whether the individual was currently taking oral hypoglycemic medication or insulin, recent illness, monthly leisuretime physical activity, BMI category, age first diagnosed diabetic, under-reporter status, and whether the individual

Table 2. Mean Ratio of Energy Intake to Estimated Basal Metabolic Rate (EI:BMR est $)$

\begin{tabular}{|c|c|c|c|}
\hline Study Group & Diabetic & Non-diabetic & Total \\
\hline \multicolumn{4}{|l|}{ Men } \\
\hline $30-59 y^{1}$ & $1.13 \pm 0.07$ & $1.49 \pm 0.02$ & $1.48 \pm 0.02$ \\
\hline$\geq 60 \mathrm{y}^{1}$ & $1.17 \pm 0.03$ & $1.31 \pm 0.02$ & $1.29 \pm 0.02$ \\
\hline \multicolumn{4}{|l|}{ Women } \\
\hline $20-29 \mathrm{y}$ & $1.26 \pm 0.32$ & $1.40 \pm 0.03$ & $1.39 \pm 0.03$ \\
\hline $30-59 y^{1}$ & $1.10 \pm 0.05$ & $1.28 \pm 0.01$ & $1.28 \pm 0.01$ \\
\hline$\geq 60 y^{1}$ & $1.00 \pm 0.03$ & $1.19 \pm 0.02$ & $1.17 \pm 0.02$ \\
\hline $20-29$ y & $1.47 \pm 0.09$ & $1.53 \pm 0.02$ & $1.53 \pm 0.02$ \\
\hline $30-59 y^{1}$ & $1.12 \pm 0.04$ & $1.39 \pm 0.01$ & $1.38 \pm 0.01$ \\
\hline$\geq 60 y^{1}$ & $1.07 \pm 0.02$ & $1.24 \pm 0.01$ & $1.22 \pm 0.01$ \\
\hline Total $\geq 20 \mathrm{y}^{1}$ & $1.10 \pm 0.02$ & $1.39 \pm 0.01$ & $1.38 \pm 0.01$ \\
\hline
\end{tabular}

${ }^{1}$ difference is statistically significant, diabetic vs. non-diabetic; $p<0.01$. 
Table 3. Demographic and Lifestyle Characteristics of Diabetic and Non-Diabetic Individuals by Under-Reporter and Adequate Reporter Status as Defined by Briefel [2]

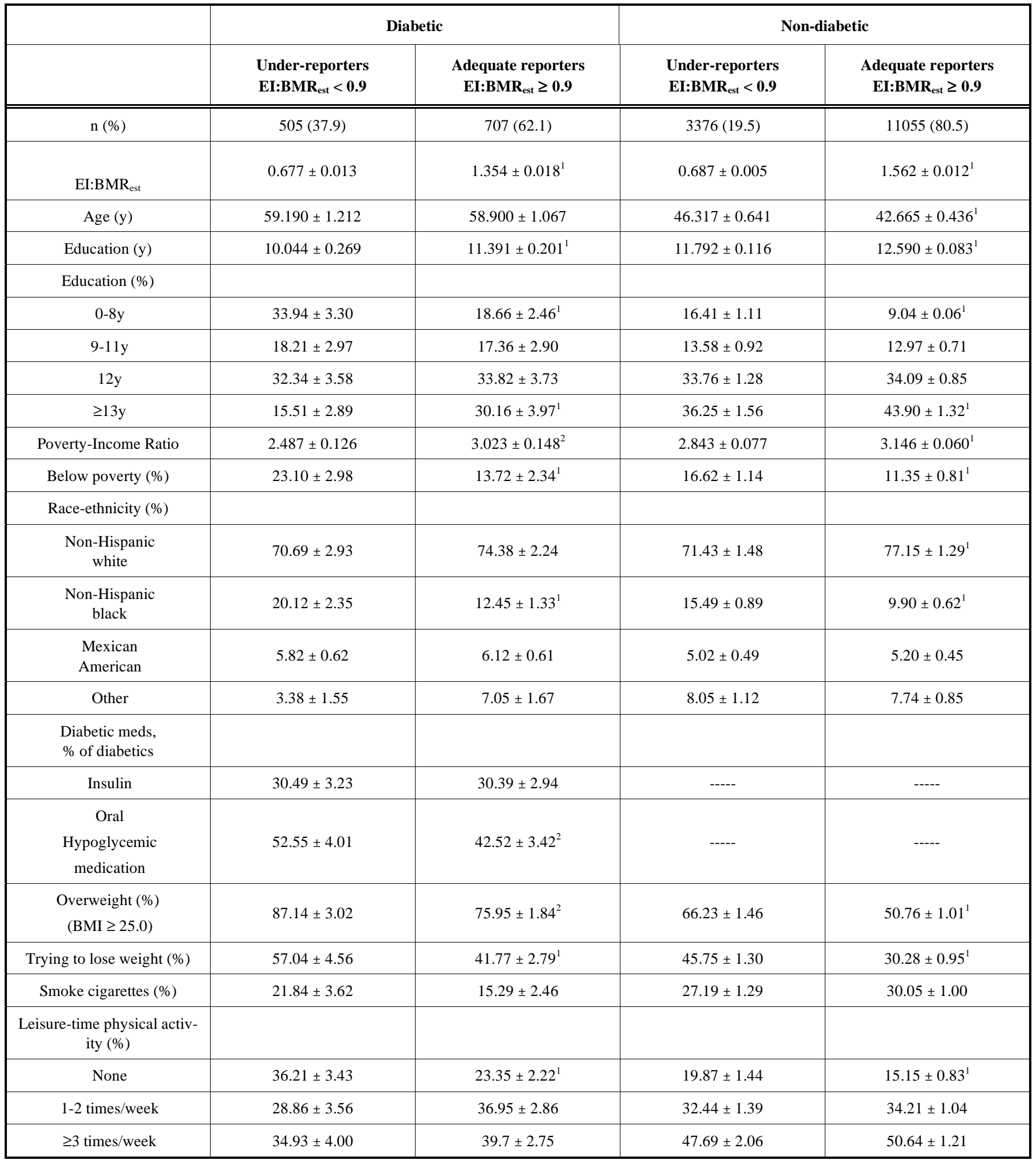

${ }^{1}$ difference is statistically significant, adequate reporters vs. under-reporters; $\mathrm{p}<0.01$. ${ }^{2}$ difference is statistically significant, adequate reporters vs. under-reporters; $\mathrm{p}<0.05$

has enough food to eat. Fasting plasma glucose level and plasma glucose levels following an oral glucose tolerance test were not included in the model because of high colinearity with the HbA1c values.

\section{RESULTS}

Data from a total of 18,513 participants, 9,705 males and 8,808 females, were analyzed. Self-identified diabetic participants numbered 1,503, of which 658 were male and 845 
female, comprising $5.2 \%$ of the total population studied (Table 1).

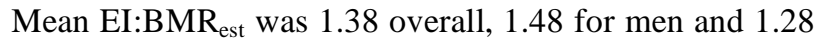
for non-pregnant women, meaning EI exceeded estimated basal metabolic rate for all participates $(48 \%$ for men and $28 \%$ for non-pregnant women). Similar to the results of Briefel et al. [2], mean EI:BMR est decreased with age and by gender. Furthermore, self-reported diabetics had lower mean $\mathrm{EI}: \mathrm{BMR}_{\mathrm{est}}$ than non-diabetic participants, 1.10 for diabetics and 1.39 for non-diabetics, $p<0.01$ (Table 2). Differences in the mean EI:BMR est were significantly different between diabetics and non-diabetics for each age group, except for subjects 20-29 years of age. Similar to Briefel et al. [2], the

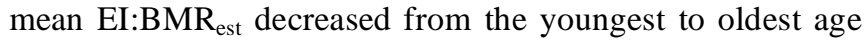
group, and from the lowest to highest BMI categories; this trend was also present within each age group.

Approximately $37.9 \%$ of self-reported diabetics and $19.5 \%$ of non-diabetics were classified as under-reporters (Table 3). For both diabetics and non-diabetics, underreporters reported a significantly lower education level, a lower income level, and were more likely to be considered below poverty and to be non-Hispanic black versus adequate reporters. Moreover, under-reporters were significantly more likely to be overweight, to report they were currently trying to lose weight, and to report zero weekly leisure-time physical activity. Compared to diabetic adequate reporters, diabetic under-reporters were more likely to currently be taking oral hypoglycemic medication. Non-diabetic under-reporters were older and a lower percent were non-Hispanic white compared to adequate reporters. A greater percent of male diabetics $(34.0 \%)$ were classified as under-reporting EI compared with male non-diabetics (14.6\%). Similarly, a greater percent of female diabetics $(41.6 \%)$ were classified as underreporting EI compared with male non-diabetics (24.2\%).

Table 4 details comparisons of mean intakes of macronutrients and selected micronutrients by level of reporting. Overall, significant differences between under-reporters and adequate reporters are present for all selected nutrients for both diabetics and non-diabetics. Under-reporters consumed approximately 4,200 kj (1000 kcal) less than adequate reporters for diabetics and 5,880 kj (1400 kcals) for nondiabetics. The percentages of energy from carbohydrate and from alcohol were not different for diabetics and nutrient intake followed differences in energy intake.

Table 4. Dietary Characteristics of Diabetic and Non-Diabetic Under-Reporters and Adequate Reporters, Based on One 24-Hour Recall as Defined by Briefel [2]

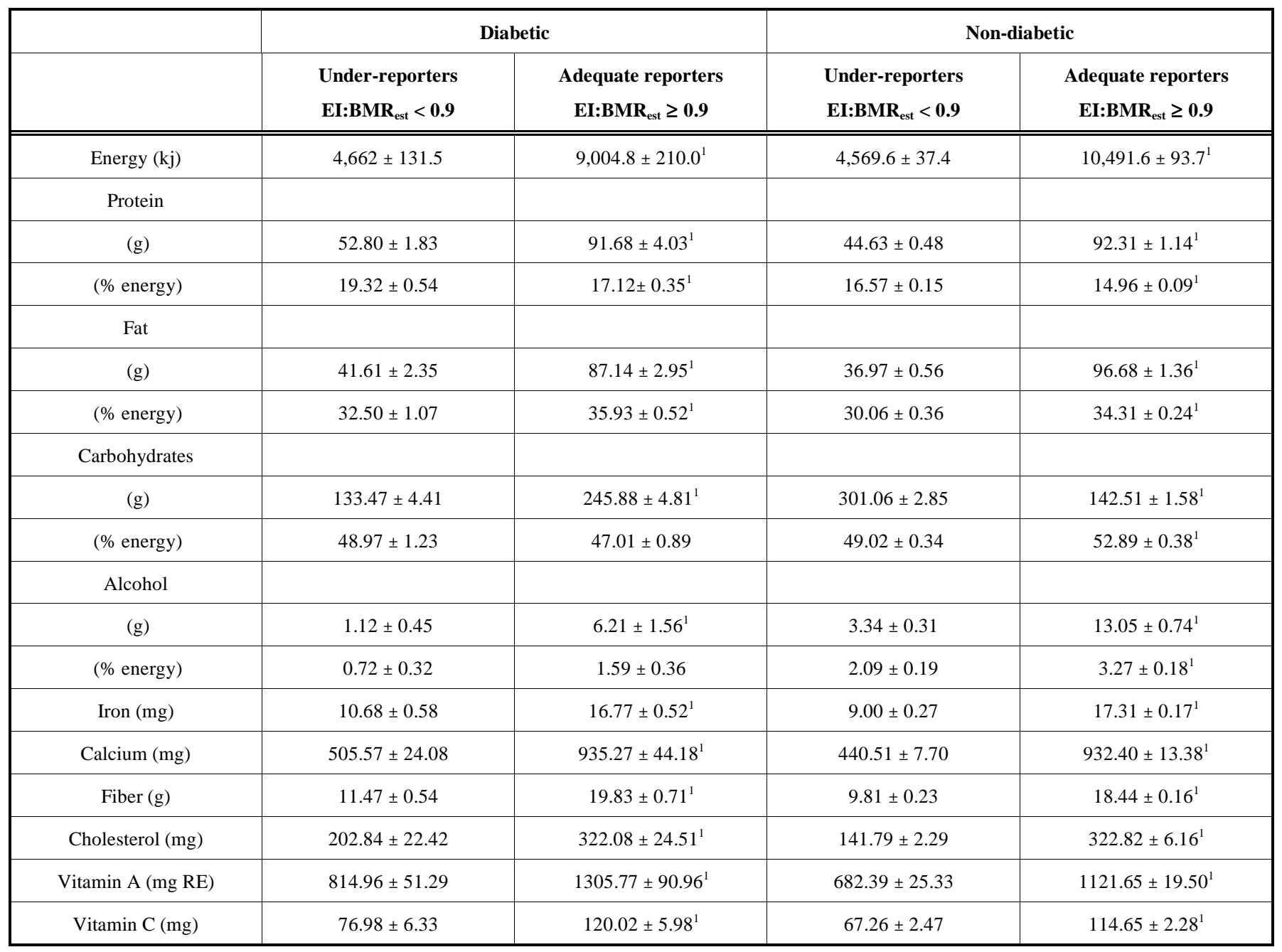

${ }^{1}$ difference is statistically significant, adequate reporters vs. under-reporters; $\mathrm{p}<0.01$. 
Differences between diabetic under-reporters and adequate reporters persisted within males and females (data not shown), but no differences were noted for non-diabetics when stratified by gender.

Men diabetics were 2.13 times as likely to under-report compared with non-diabetics after adjusting for potential confounding factors. Diabetic women were 3.39 times as likely to under-report compared with non-diabetic women after adjusting for potential confounding factors (Table $\mathbf{5}$ ).

Among self-identified diabetics, a series of analyses were conducted to determine how various factors such as diabetes medications and under-reporter status were associated with

Table 5. Results of Multiple Logistic Regression Analyses to Compare Under-Reporting Between Diabetics and Non-Diabetics Stratified by Gender Using the Briefel Standard [2]

\begin{tabular}{|c|c|c|c|}
\hline Variable & $\mathbf{p}$ & OR & $95 \% \mathrm{CI}$ \\
\hline Diabetic & $<0.001$ & 2.126 & $1.496-3.021$ \\
\hline Age (y) & $<0.001$ & 1.017 & $1.010-1.025$ \\
\hline Education, y completed & 0.003 & 0.940 & $0.903-0.977$ \\
\hline \multicolumn{4}{|l|}{ Intake level, vs. more than usual } \\
\hline "Usual" intake & 0.039 & 4.594 & $1.081-19.520$ \\
\hline Less than "usual" intake & 0.004 & 8.750 & $2.088-36.670$ \\
\hline Overweight (BMI $\geq 25.0$ ) & $<0.001$ & 1.596 & $1.254-2.030$ \\
\hline Currently trying to lose weight & 0.006 & 1.495 & $1.127-1.985$ \\
\hline Equally as active & 0.004 & 1.495 & $1.147-1.950$ \\
\hline Born in Mexico (vs. USA) & $<0.001$ & 0.455 & $0.315-0.658$ \\
\hline Below poverty & $<0.001$ & 2.085 & $1.568-2.772$ \\
\hline \# colds or flue in past 4 weeks & 0.033 & 0.848 & $0.729-0.986$ \\
\hline Ever smoke cigars or pipes & 0.027 & 0.759 & $0.595-0.967$ \\
\hline $12+$ alcoholic drinks in past year & 0.034 & 0.764 & $0.596-0.979$ \\
\hline Recall day (weekend vs. weekday) & 0.050 & 0.717 & $0.514-1.000$ \\
\hline \multicolumn{4}{|l|}{ Women } \\
\hline Less than "usual" intake & $<0.001$ & 8.684 & $3.798-19.855$ \\
\hline Overweight (BMI $\geq 25.0$ ) & $<0.001$ & 1.761 & $1.415-2.192$ \\
\hline Currently trying to lose weight & $<0.001$ & 1.643 & $1.337-2.018$ \\
\hline \multicolumn{4}{|c|}{ Weight status, desire to change vs. desire to weight more } \\
\hline Desire to weigh less (vs. more) & 0.006 & 1.850 & $1.201-2.851$ \\
\hline Desire to weigh same (vs. more) & 0.014 & 1.792 & $1.129-2.845$ \\
\hline Taken antihistamines in past 2 days & 0.033 & 0.752 & $0.579-0.977$ \\
\hline Ever consume $12+$ alcoholic drinks & 0.003 & 0.793 & $0.682-0.922$ \\
\hline Interaction of diabetic * year of education & 0.053 & 0.931 & $0.866-1.001$ \\
\hline
\end{tabular}


HbA1c levels. Under-reporting status and BMI were not associated with the $\mathrm{HbA} 1 \mathrm{c}$ values after adjusting for diabetic medication in both men and women (Table 6). For men, age was negatively associated with $\mathrm{HbA1c}$ but a history of smoking was positively associated with HbA1c. For women, age, having smoked cigars, percent of energy from alcohol, and having been told about retinopathy were associated with HbA1c values.

\section{CONCLUSIONS}

Briefel explored under-reporting using 24-hour dietary recalls in the first phase of NHANES III to determine if procedures for collecting data had improved since NHANES I and NHANES II [2]. Although procedures appeared to improve, as mean EI:BMR est values were higher in NHANES III than in NHANES I and NHANES II, and under-reporting was still present. This current research builds on previous work to explore under-reporting using data from both phases of NHANES III and to investigate if an association exists between self-identified diabetic status and under-reporting of EI. Furthermore, the relative impact of under-reporting on long-term glycemic control among self-identified diabetics was explored.

Diabetic males were 2.1 times as likely to under-report compared with non-diabetic males, using an EI:BMR est $<0.9$ to classify under-reporters. Using the same cutoff value, diabetic females were 3.4 times as likely to under-report com- pared with non-diabetic females. These effects are seen after adjusting for factors that would result in lower reported energy intake, such as attempting to lose weight, "usual" intake level and physical activity level.

The association of diabetic status with EI:BMR $\mathrm{est}_{\text {can }}$ be quantified, though interpretation of results is considerably less straightforward. For both men and women, diabetic status was significantly associated with a decreased $\mathrm{EI}: \mathrm{BMR}_{\mathrm{est}}$ ratio, indicating that diabetics appear more likely to under-report than non-diabetics. While a significant factor, the relative contribution of diabetic status to the overall EI:BMR $\mathrm{est}_{\text {r }}$ ratio was small, accounting for roughly $1 \%$ of the total $R^{2}$.

These results are similar to other studies. Using the first phase of the NHANES III dataset, Briefel found significant

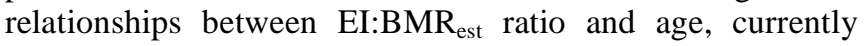
trying to lose weight, education, smoking status, and "usual" intake [2]. Alemzadeh et al. [18] studying a small group of young, type 1 diabetes patients (24 males, 20 females, mean age $13.2 \pm 4.5$ ), compared type 1 diabetes with age and sex matched non-diabetic controls and found no significant association between diabetic status and under-reporting. Examining solely type 1 diabetics was a shortcoming of Alemzadeh's work [18] making comparison with the present results difficult. NHANES III only provides data on diabetic status at interview, without differentiating between type 1 and type 2 diabetes. If any differential effects exist between

Table 6. Results of Multiple Linear Regression Analyses to Determine the Association Between Hemoglobin A1c Levels and Under-Reporting in Diabetic Men and Women, Third National Health and Nutrition Examination Survey

\begin{tabular}{|c|c|c|c|}
\hline Variable & Coefficient & $\mathbf{t}$ & $\mathbf{p}$ \\
\hline Currently using insulin & $1.20(0.33)$ & 3.6 & 0.001 \\
\hline $\begin{array}{l}\text { Currently taking oral hypoglycemic } \\
\text { medication }\end{array}$ & $0.88(0.37)$ & 2.4 & 0.05 \\
\hline Ever smoked cigarettes & $0.65(0.26)$ & 2.5 & 0.05 \\
\hline Constant & 7.58 & & \\
\hline \multicolumn{4}{|l|}{$\mathrm{F}=5.7 ; \mathrm{p}<0.001 ; \mathrm{R} 2=0.10$} \\
\hline \multicolumn{4}{|l|}{ Women } \\
\hline Ever been told about retinopathy & $0.66(0.27)$ & 2.4 & 0.05 \\
\hline Ever smoked cigars & $1.67(0.25)$ & 6.7 & 0.001 \\
\hline Percent of energy from alcohol & $-0.07(0.02)$ & -4.4 & 0.001 \\
\hline Age $30-39$ vs. $20-29$ (y) & $-1.85(0.47)$ & -3.9 & 0.001 \\
\hline Age $\geq 60$ vs. $20-29(\mathrm{y})$ & $-2.37(0.47)$ & -5.0 & 0.001 \\
\hline Constant & 8.52 & & \\
\hline
\end{tabular}

$\mathrm{F}=35.3 ; \mathrm{p}<0.001 ; \mathrm{R} 2=0.27$. 
types 1 and type 2, diabetes they cannot be detected and described.

A continuing problem for these analyses is that assessing dietary intake bias is done by comparing reported EI with estimated BMR. In effect, an assessment of bias is accomplished using an estimate of "truth" that is itself biased. Without knowing a person's exact energy expenditure, it is difficult to accurately establish under-reporter status. While use of a low cut point may classify those who grossly underreport correctly, those with higher true energy expenditures

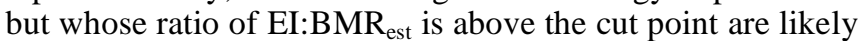
to be classified as adequate reporters. Adjustment during analysis using reported number of activities and "usual" intake may not be sufficient to account for the misclassification. These data assess only leisure-time physical activity and do not include physical activity at work nor duration of the activity.

An additional problem is that estimated BMR does not adjust for differences in actual energy expenditure due to illness or chronic disease status. Diabetic patients not maintaining proper glycemic control actually have greater energy expenditure levels than non-diabetics [23] and with after treatment with insulin or oral hypoglycemic medication, energy expenditure reverts toward normal. Such effects have been noted for both type 1 [24] and type 2 [25] diabetic patients. Thus, the magnitude of the relationship between dietary under-reporting and diabetic status may actually be greater than was observed. Methods other than $\mathrm{BMR}_{\mathrm{est}}$, such as whole-body calorimetry [6], doubly-labeled water [6-8], and urinary biomarkers [9-10], are likely to more accurately represent energy expenditure. However, these methods are expensive and impractical for large surveys.

Another concern is the use of $\mathrm{BMR}_{\text {est }}$ as the denominator of the dependent variable in regression models that adjust for age and BMI or height and weight. This is a potential problem as BMR $_{\text {est }}$ was calculated using age-specific formulas that incorporate height and weight, and the resulting model may not be statistically sound. Other studies that have also used the $\mathrm{BMR}_{\text {est }}$, include work performed using the first phase of NHANES III [2], the analysis of the Norwegian NORKOST survey by Johansson [3] and Pryer's work using the Dietary and Nutritional Survey of British Adults [4]. Similar relationships between dietary under-reporting, age and body mass index are noted in all these studies with under-reporting of energy intake increasing with age and more likely to occur in overweight or obese participants. Further, calculated $\mathrm{BMR}_{\mathrm{est}}$ is not being predicted in these analyses, rather the degree of under-reporting is being measured.

More research must take place to understand how the use of imperfect equations to predict $\mathrm{BMR}_{\mathrm{est}}$ affects assessment of under-reporting. Moreover, as the approach employed in this research is common, exploration must occur on how analyses are hampered when dependent variables are formulated, at least in part, when using factors that are also included as independent factors.

Monitoring HbA1c has become a common tool for assessing glycemic control in diabetic patients [21]. The rationale for utilizing HbA1c includes the measure's ability to objectively assess blood glucose levels during the preceding two to three months [22]. As excessive blood glucose over long periods can lead to serious microvascular complications, a simple, objective measure such as HbA1c is of considerable utility to clinicians and patients. Indeed, the Diabetes Control and Complications Trial (DCCT) has clinically validated the measure, showing that improved glycemic control can reduce the risk of microvascular complications [26] and lead to improved quality of life in diabetic patients.

Understanding the association between dietary underreporting and long-term glycemic control could be a boon to diabetic patients and clinicians alike, allowing more effective dietary recommendations to be formulated. However, in this study long-term glycemic control was not found to be significantly associated with dietary under-reporting.

Under-reporting was not associated with increased $\mathrm{HbA1c}$ and the major behavioral contributor to HbA1c values was whether a diabetic was taking insulin or oral hypoglycemic drugs. Taking hypoglycemic medication probably lowered the HbA1c values in diabetics who under-reported.

Under-reporting energy intake can complicate the understanding of diet-disease relationships, potentially altering disease management. Furthermore, under-reporting can bias the association between diet and disease toward the null when it occurs in diseased patients. This may become especially important when trying to determine the effect of diet on diseases associated with diabetes, such as cardiovascular disease, neuropathies, and retinopathy. For example, if diabetics are at greater risk for cardiovascular disease, but at the same time have greater under-reporting, the overall association between diet and cardiovascular disease will be biased toward the null due to the greater under reporting in diabetics. A better understanding of which populations are more likely to under-report has the potential to improve the analyses between diet and diseases.

\section{ABBREVIATIONS}

$\begin{array}{ll}\mathrm{BMR}_{\mathrm{est}} & \text { Estimated basal metabolic rate } \\ \mathrm{EI} & =\text { Energy intake } \\ \text { EI:BMR } & =\text { Ratio of energy intake to estimated basal } \\ & \text { metabolic rate } \\ \text { HbA1c } & \text { Hemoglobin A1c } \\ \text { NHANES III = } & \text { Third National Health and Nutrition Ex- } \\ & \text { amination Survey }\end{array}$

PSU $\quad=$ Population sampling unit

\section{ACKNOWLEDGEMENTS}

This project was supported by a grant from the National Institutes of Health, National Institute of Diabetes, Digestive and Kidney Diseases, Grant RO1-DK48906.

\section{REFERENCES}

[1] Black AE, Goldberg GR, Jebb SA, et al. Critical evaluation of energy intake using fundamental principles of energy physiology. 2. Evaluating the results of published surveys. Eur J Clin Nutr 1991; 45: 583-99.

[2] Briefel RR, Sempos CT, McDowell MA, et al. Dietary methods research in the third National Health and Nutrition Examination Survey: under-reporting of energy intake. Am J Clin Nutr 1997; 65(Suppl): 1203S-9S.

[3] Johansson L, Solvoll K, Bjørnebee G-EA, Drevon CA. Under- and overreporting of energy intake related to weight status and lifestyle in a nationwide sample. Am J Clin Nutr 1998; 68: 266-74. 
[4] Pryer JA, Vrijheid M, Nichols R, et al. Who are the 'low energy reporters' in the Dietary and Nutritional Survey of British Adults? Int J Epidemiol 1997; 26: 146-154.

[5] Samaras K, Kelly PJ, Campbell LV. Dietary under-reporting is prevalent in middleaged British women and is not related to adiposity (percentage body fat). Int J Obesity 1999; 23: 881-8.

[6] Goldberg GR, Black AE, Jebb SA, et al. Critical evaluation of energy intake using fundamental principles of energy physiology. 1. Derivation of cut-off limits to identify under-recording. Eur J Clin Nutr 1991; 45: 569-81.

[7] Kaczkowski CH, Jones PJH, Feng J, Bayley HS. Four-day multimedia diet records underestimate energy needs in middle-aged and elderly women as determined by doubly-labeled water. J Nutr 2000; 130: 802-5.

[8] Johnson RK, Soultanakis RP, Matthews DE. Literacy and body fatness are associated with under-reporting of energy intake in US low-income women using the multiple-pass 24-hour recall: a doubly labeled water study. J Am Diet Assoc 1998; 98: 1136-40.

[9] Heitmann BL, Lissner L. Dietary under-reporting by obese individuals: is it specific or non-specific? BMJ 1995; 311: 986-9.

[10] Zhang J, Temme EHM, Sasaki S, Kesteloot H. Under- and overreporting of energy intake using urinary cations as biomarkers: relation to body mass index. Am J Epidemiol 2000; 152: 453-62.

[11] Johnson RK, Goran MI, Poehlman ET. Correlates of over- and under-reporting of energy intake in healthy older men and women. Am J Clin Nutr 1994; 59: 1286-90.

[12] Mokdad AH, Ford ES, Bowman BA, et al. Diabetes trends in the US: 1990-1998. Diabetes Care 2000; 23: 1278-83.

[13] Harris MI, Goldstein DE, Flegal KM, et al. Prevalence of diabetes, impaired fasting glucose, and impaired glucose tolerance in US adults. Diabetes Care 1998; 21: 518-25.

[14] Harris MI. Diabetes in America: epidemiology and scope of the problem. Diabetes Care 1998; 21(Suppl 3): C11-C14.

[15] Venkat Narayan KM, Gregg EW, Fagot-Campagna A, et al. Diabetes - a common, growing, serious, costly, and potentially preventable public health problem. Diabetes Res Clin Pract 2000; 50(Suppl): S77-S84.
[16] de Alva ML. The burden of diabetes: the patient's perspective. Diabetes Care 1998; 21(Suppl 3): C29.

[17] Rubin RJ, Altman WM, Mendelson DN. Health care expenditures for people with diabetes mellitus, 1992. J Clin Endocrinol Metab 1994; 78: 809A-F.

[18] Alemzadeh R, Goldberg R, Fort P, et al. Reported dietary intakes of patients with insulin-dependent diabetes mellitus: limitations of dietary recall. Nutrition 1992; 8: 87-93.

[19] US Department of Health and Human Services (DHHS). National Center for Health Statistics. Third National Health and Nutrition Examination, 1998-1994, NHANES III Laboratory Data File (CDROM). Public Use Data File Documentation Number 76200. Hyattsville, MD: Centers for Disease Control and Prevention, 1996.

[20] Schofield WN. Predicting basal metabolic rate, new standards and review of previous work. Hum Nutr Clin Nutr 1985; 39C(Suppl 1): $5-41$.

[21] Kilpatrick ES, Maylor PW, Keevil BG. Biological variation of glycated hemoglobin: implications for diabetes screening and monitoring. Diabetes Care 1998; 21: 261-4.

[22] Goldstein DE, Little RR, Wiedmeyer H-M, et al. Glycated hemoglobin: methodologies and clinical applications. Clin Chem 1986; 32: B64-B70.

[23] Pi-Sunyer FX. Overnutrition and undernutrition as modifiers of metabolic processes in disease states. Am J Clin Nutr 2000; 72(Suppl): 533S-7S

[24] Nair KS, Halliday D, Garrow JS. Increased energy expenditure in poorly controlled type 1 (insulin-dependent) diabetic patients. Diabetalogia 1984; 27: 13-16.

[25] Bogardus C, Taskinen M-R, Zawakzki J, et al. Increased resting metabolic rates in obese subjects with non-insulin dependent diabetes mellitus and the effect of sulfonylurea therapy. Diabetes 1986; 35: $1-5$.

[26] The DCCT Research Group: The effect of intensive treatment of diabetes on the development and progression of long-term complications in insulin-dependent diabetes mellitus. N Engl J Med 1993; 329: $977-86$

(C) Broyles et al.; Licensee Bentham Open.

This is an open access article distributed under the terms of the Creative Commons Attribution License (http://creativecommons.org/licenses/by/2.5/), which permits unrestrictive use, distribution, and reproduction in any medium, provided the original work is properly cited. 